\title{
INTERNAL NONLOCAL AND INTEGRAL CONDITION PROBLEMS OF THE DIFFERENTIAL EQUATION $x^{\prime}=f\left(t, x, x^{\prime}\right)$
}

\author{
A. M. A. EL-SAYED ${ }^{1}$, E. M. HAMDALLAH ${ }^{2}$ AND KH. W. ELKADEKY ${ }^{3}$
}

ABSTRACT. In this work, we are concerned with the existence of at least one absolutely continuous solution of the Cauchy problem for the differential equation $x^{\prime}=f\left(t, x, x^{\prime}\right), t \in(0,1)$ with the internal nonlocal condition $\sum_{k=1}^{m} a_{k} x\left(\tau_{k}\right)=x_{o}, \tau_{k} \in(c, d) \subseteq(0,1)$. The problem of the integral condition $\int_{c}^{d} x(s) d g(s)=x_{o}$ will be considered.

\section{INTRODUCTION}

Problems with non-local conditions have been extensively studied by several authors in the last two decades. The reader is referred to [प]- [G] and [Q] - [प5] and references therein.

Here we are consisted with the nonlocal problem

$$
\begin{aligned}
\frac{d x(t)}{d t} & =f\left(t, x(t), \frac{d x(t)}{d t}\right), \text { a.e }, t \in(0,1), \\
\sum_{k=1}^{m} a_{k} x\left(\tau_{k}\right) & =x_{o}, \sum_{k=1}^{m} a_{k} \neq 0 \text { and } \tau_{k} \in(c, d) \subseteq(0,1) .
\end{aligned}
$$

The existence of at least one solution $x \in A C[0,1]$ will be studied when the function $f$ is measurable in $t \in[0,1]$,for any $\left(u_{1}, u_{2}\right) \in R^{2}$ and continuous in $\left(u_{1}, u_{2}\right) \in R^{2}$, for $t \in[0,1]$. As a consequence of our result, the problem of the

Date: Received: 1 Jan, 2011; Revised: 20 April, 2011.

*Corresponding author

(c) 2011 N.A.G.

2000 Mathematics Subject Classification. Primary 26A33; Secondary 34A12, 34A40.

Key words and phrases. Nonlocal conditions, integral condition, existence of solution, fixedpoint theorem. 
differential equation $(\mathbb{\square} \mathbb{d})$ with integral condition

$$
\int_{c}^{d} x(s) d g(s)=x_{o},
$$

where $g$ is a nondecreasing function, will be studied.

It must be noticed that the nonlocal condition (․? ) and the integral condition (ㅍ.3) are more general than the following ones

$$
\begin{gathered}
x(\tau)=x_{o}, \tau \in(c, d), \\
\sum_{k=1}^{m} a_{k} x\left(\tau_{k}\right)=0, \tau_{k} \in(a, c),
\end{gathered}
$$

and

$$
\int_{a}^{c} x(s) d g(s)=0 .
$$

The following theorems will be needed.

Theorem (Kolmogorov Compactness Criterion) see [ []$]$ Let $\Omega \subseteq L^{P}(0,1), 1 \leq P<\infty$. If

(i) $\Omega$ is bounded $L^{p}(0,1)$,

(ii) $x_{h} \rightarrow x$ as $h \rightarrow 0$ uniformly with respect to $x \in \Omega$, then $\Omega$ is relatively compact in $L^{P}(0,1)$, where

$$
x_{h}(t)=\frac{1}{h} \int_{t}^{t+h} x(s) d s .
$$

Theorem (Schauder) see[प्2]

Let $U$ be a convex subset of a Banach space $X$, and $T: U \rightarrow U$ is compact, continuous map. Then $T$ has at least one fixed point in $U$.

\section{EXISTENCE OF SOLUTION}

The following Lemma gives the integral equation representation for the nonlo-

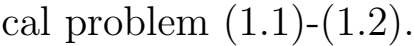

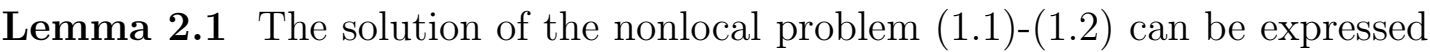
by the integral equation

$$
x(t)=a x_{0}-a \sum_{k=1}^{m} a_{k} \int_{0}^{\tau_{k}} y(s) d s+\int_{0}^{t} y(s) d s
$$

where $y$ is the solution of the functional integral equation

$$
y(t)=f\left(t, a x_{0}-a \sum_{k=1}^{m} a_{k} \int_{0}^{\tau_{k}} y(s) d s+\int_{0}^{t} y(s) d s, y(t)\right), t \in(0,1) .
$$

Proof. Let $\frac{d x(t)}{d t}=y(t)$ in equation (1), then

$$
y(t)=f\left(t, x(0)+\int_{0}^{t} y(s) d s, y(t)\right)
$$


where

$$
x(t)=x(0)+\int_{0}^{t} y(s) d s .
$$

Let $t=\tau_{k}$ in $(\mathbb{2} \cdot \mathbf{U})$, we obtain

$$
x\left(\tau_{k}\right)=x(0)+\int_{0}^{\tau_{k}} y(s) d s
$$

and

$$
\sum_{k=1}^{m} a_{k} x\left(\tau_{k}\right)=\sum_{k=1}^{m} a_{k} x(0)+\sum_{k=1}^{m} a_{k} \int_{0}^{\tau_{k}} y(s) d s .
$$

Substitute from (2) into ( $\overline{2.5})$, we get

$$
x_{0}=\sum_{k=1}^{m} a_{k} x(0)+\sum_{k=1}^{m} a_{k} \int_{0}^{\tau_{k}} y(s) d s
$$

and

$$
x(0)=a\left(x_{0}-\sum_{k=1}^{m} a_{k} \int_{0}^{\tau_{k}} y(s) d s\right)
$$

where $a=\left(\sum_{k=1}^{m} a_{k}\right)^{-1}$.

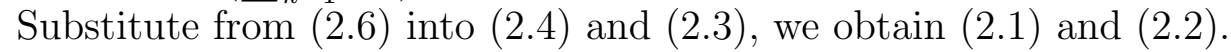

Consider the functional integral equation ( $(2 \cdot)$ with the following assumptions

(i) $f:[0,1] \times R^{2} \rightarrow R^{+}$is measurable in $t \in[0,1]$ for any $\left(u_{1}, u_{2}\right) \in R^{2}$ and continuous in $\left(u_{1}, u_{2}\right) \in R^{2}$ for almost all $t \in[0,1]$.

(ii) There exists a function $a \in L_{1}[0,1]$ and two constants $b_{i}>0, i=1,2$ such that

$$
\left|f\left(t, u_{1}, u_{2}\right)\right| \leq|a(t)|+\sum_{i=1}^{2} b_{i}\left|u_{i}\right|, \forall\left(t, u_{1}, u_{2}\right) \in[0,1] \times R^{2} .
$$

(iii)

$$
\left(2 b_{1}+b_{2}\right)<1 .
$$

Now we have the following theorem.

Theorem 2.1 Assume that the assumptions (i) - (iii) are satisfied. Then the functional integral equation ( $(\mathbb{2} \cdot)$ has at least one solution $y \in L_{1}(0,1)$.

Proof. Let $y \in B_{r} \subset L^{1}, B_{r}=\left\{y:\|y\|_{L_{1}} \leq r, r>0\right\}, r=\frac{\|a\|+a b_{1} x_{0}}{1-\left(2 b_{1}+b_{2}\right)}$.

Clearly $B_{r}$ is nonempty, convex and closed .

Define the operator $H$ by

$$
H y(t)=f\left(t, a x_{0}-a \sum_{k=1}^{m} a_{k} \int_{0}^{\tau_{k}} y(s) d s+\int_{0}^{t} y(s) d s, y(t)\right), t \in(0,1) .
$$


From assumptions (i) and (iii), we obtain

$$
\begin{aligned}
\|H y\|_{L_{1}} & =\int_{0}^{1}|(H y)(t)| d t \\
& =\int_{0}^{1}\left|f\left(t, a x_{0}-a \sum_{k=1}^{m} a_{k} \int_{0}^{\tau_{k}} y(s) d s+\int_{0}^{t} y(s) d s, y(t)\right)\right| d t \\
& \leq \int_{0}^{1}\left(|a(t)|+b_{1}\left|a x_{o}-a \sum_{k=1}^{m} a_{k} \int_{0}^{\tau_{k}} y(s) d s+\int_{0}^{t} y(s) d s\right|+b_{2}|y(t)|\right) d t \\
& \leq \int_{0}^{1}\left(|a(t)| d t+\int_{0}^{1} b_{1} a x_{o} d t+a b_{1} \sum_{k=1}^{m} a_{k} \int_{0}^{1} \int_{0}^{\tau_{k}}|y(s)| d s d t\right. \\
& \left.+b_{1} \int_{0}^{1} \int_{0}^{t}|y(s)| d s d t+b_{2} \int_{o}^{1}|y(t)|\right) d t \\
& \leq\|a\|+a b_{1} x_{o}+b_{1}\|y\|+b_{1}\|y\|+b_{2}|| y \| \\
& \leq\|a\|+b_{1} a x_{0}+\left(2 b_{1}+b_{2}\right)\|y\| \leq r .
\end{aligned}
$$

Then $\|H y\|_{L_{1}} \leq r$, which implies that the operator $H$ maps $B_{r}$ into itself. Assumption (i) implies that $H$ is continuous.

Now, let $\Omega$ be a bounded subset of $B_{r}$, therefore $H(\Omega)$ is bounded in $L_{1}(0,1)$, i.e condition (i) of Kolmogorav compactness criterion is satisfied, it remains to show $(H y)_{h} \rightarrow(H y)$, in $L_{1}(0,1]$.

Let $y \in \Omega \subset L_{1}(0,1)$, then we have the following

$$
\begin{aligned}
\left\|(H y)_{h}-(H y)\right\|_{L_{1}} & =\int_{0}^{1}\left|(H y)_{h}(t)-(H y)(t)\right| d t \\
& =\int_{0}^{1}\left|\frac{1}{h} \int_{t}^{t+h}(H y)(s) d s-(H y)(t)\right| d t \\
& \leq \int_{0}^{1}\left(\frac{1}{h} \int_{t}^{t+h}|(H y)(s)-(H y)(t)| d s\right) d t \\
& \leq \int_{0}^{1} \frac{1}{h} \int_{t}^{t+h} \mid f\left(s, a x_{0}-a \sum_{k=1}^{m} a_{k} \int_{0}^{s_{k}} y(\tau) d \tau+\int_{0}^{s} y(\tau) d \tau, y(s)\right) \\
& -f\left(t, a x_{0}-a \sum_{k=1}^{m} a_{k} \int_{0}^{\tau_{k}} y(s) d s+\int_{0}^{t} y(s) d s, y(t)\right) \mid d s d t .
\end{aligned}
$$

Since $y \in \Omega \subset L_{1}$, and (assumption (ii) implies that) $f \in L_{1}[0,1]$, it follows that

$$
\frac{1}{h} \int_{t}^{t+h} \mid f\left(s, a x_{0}-a \sum_{k=1}^{m} a_{k} \int_{0}^{s_{k}} y(\tau) d \tau+\int_{0}^{s} y(\tau) d \tau, y(s)\right)
$$


$-f\left(t, a x_{0}-a \sum_{k=1}^{m} a_{k} \int_{0}^{\tau_{k}} y(s) d s+\int_{0}^{t} y(s) d s, y(t)\right) \mid d s \rightarrow 0$ as $h \rightarrow 0, t \in(0,1)$.

Hence $(H y)_{h} \rightarrow(H y)$, uniformly as $h \rightarrow 0$.

Then by Kolmogorav compactness criterion, $H(\Omega)$ is relatively compact.

That is $H$ has a fixed point in $B_{r}$, then there exist at least one solution $y \in L_{1}(0,1)$ of the functional equation ([2.3).

Now, consider the nonlocal problem $(\mathbb{\square} \mathbb{C})-(\mathbb{L} 2)$.

Theorem 2.2 Let the assumptions of Theorem 2.1 are satisfied. Then the

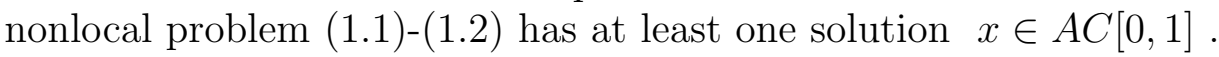

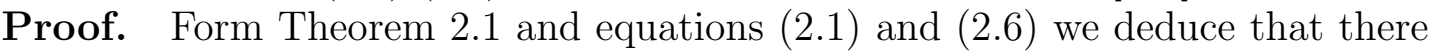
exist at least one solution $x \in A C[0,1]$ of equation ( $[\mathbb{Z}]$ ) where

$$
x(0)=\lim _{t \rightarrow 0} x(t)=a x_{0}-a \sum_{k=1}^{m} a_{k} \int_{0}^{\tau_{k}} y(s) d s
$$

and

$$
x(1)=\lim _{t \rightarrow 1} x(t)=a x_{0}-a \sum_{k=1}^{m} a_{k} \int_{0}^{\tau_{k}} y(s) d s+\int_{0}^{1} y(s) d s
$$

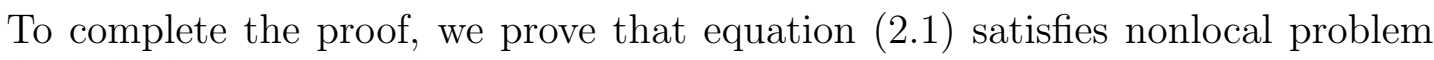

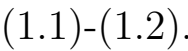

Differentiating ([2]), we get

$$
\frac{d x}{d t}=y(t)=f\left(t, x(t), \frac{d x}{d t}\right)
$$

Let $t=\tau_{k}$ in $(\mathbb{E . T})$, we get

$$
\begin{aligned}
x\left(\tau_{k}\right)= & a x_{0}-a \sum_{k=1}^{m} a_{k} \int_{0}^{\tau_{k}} y(s) d s+\int_{0}^{\tau_{k}} y(s) d s \\
& =a x_{0}+\left(1-a \sum_{k=1}^{m} a_{k}\right) \int_{0}^{\tau_{k}} y(s) d s .
\end{aligned}
$$

Then

$$
\sum_{k=1}^{m} a_{k} x\left(t_{k}\right)=\sum_{k=1}^{m} a_{k} a x_{0}+\sum_{k=1}^{m} a_{k}\left(1-a \sum_{k=1}^{m} a_{k}\right) \int_{0}^{\tau_{k}} y(s) d s=x_{o} .
$$

This complete the proof of the equivalent between the nonlocal problem $(\mathbb{\mathbb { D }} \mathbb{\text { D }}$ (ㅁ.2) and the integral equation (एत) .

This implies that there exist at least one solution $x \in A C[0,1]$ of the nonlocal

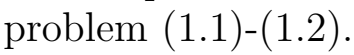




\section{Nonlocal integral CONDition}

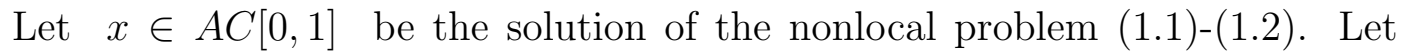
$a_{k}=g\left(t_{k}\right)-g\left(t_{k-1}\right), g$ is a nondecreasing function, $\tau_{k} \in\left(t_{k-1}, t_{k}\right), c=t_{0}<$ $t_{1}<t_{2}, \ldots<t_{n}=d$, then the nonlocal condition (마) will be

$$
\sum_{k=1}^{m}\left(g\left(t_{k}\right)-g\left(t_{k-1}\right)\right) x\left(\tau_{k}\right)=x_{o} .
$$

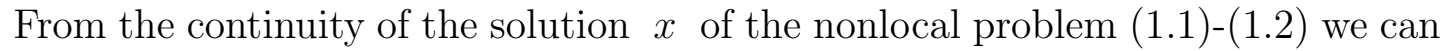
obtain

$$
\lim _{m \rightarrow \infty} \sum_{k=1}^{m}\left(g\left(t_{k}\right)-g\left(t_{k-1}\right)\right) x\left(\tau_{k}\right)=\int_{c}^{d} x(s) d g(s) .
$$

and the nonlocal condition (2) transformed to the integral one

$$
\int_{c}^{d} x(s) d g(s)=x_{o}
$$

Now, we have the following Theorem

Theorem 3.1 Let the assumptions of Theorem 2.1 are satisfied. Then there exists at least one solution $x \in A C[0,1]$ of the nonlocal problem with integral condition,

$$
\begin{gathered}
\frac{d x(t)}{d t}=f\left(t, x(t), \frac{d x(t)}{d t}\right), \text { a.e }, t \in(0,1], \\
\int_{c}^{d} x(s) d g(s)=x_{o} .
\end{gathered}
$$

\section{REFERENCES}

[1] M. Benchohra, E.P. Gatsori and S.K. Ntouyas, Existence results for seme-linear integrodifferential inclusions with nonlocal conditions. Rocky Mountain J. Mat. Vol. 34, No. 3, Fall 2004

[2] M. Benchohra, S. Hamani, S. Ntouyas, Boundary value problems for differential equations with fractional order and nonlocal conditions, Nonlinear Analysis Vol.71 (2009) 23912396

[3] Boucherif, A. First-order differential inclusions with nonlocal initial conditions, Applied Mathematics Letters Vol.15 (2002) 409-414.

[4] A. Boucherif, Nonlocal Cauchy problems for first-order multivalued differential equations, Electronic Journal of Differential Equations Vol. 2002 (2002), No. 47, pp. 1-9.

[5] Boucherif, A and Precup, R. On The nonlocal Initial Value Problem for First Order Differential Equations, Fixed Point Theory,Volume 4,No 2,(2003)205-212.

[6] Boucherif, A. Semilinear evolution inclusions with nonlocal conditions, Applied Mathematics Letters Vol.22 (2009) 1145-1149.

[7] Curtain, R. F. and Pritchard, A. J. Functional Analysis in modern, Applied Mathematics Academic Press (1977).

[8] Dugundji, J. and Grans, A. Fixed Point Theory, Monografie Mathematyczne, PWN, Warsaw (1963).

[9] El-Sayed, A. M. A. and Abd El-Salam, Sh. A. On the stability of a fractional order differential equation with nonlocal initial condtion, EJQTDE, 29(2008)1-8. 
[10] El-Sayed, A.M.A and Elkadeky, Kh. W. Caratheodory theorem for a nonlocal problem of the differential equation $x^{\prime}=f\left(t, x^{\prime}\right)$, Alexandria j. of Math. Vol. 1 No. 2 (2010) In press.

[11] Gatsori. E, Ntouyas. S. K, and Sficas. Y.G. On a nonlocal cauchy problem for differential inclusions, Abstract and Applied Analysis (2004) 425-434.

[12] Goebel. K and Kirk W. A. Topics in Metric Fixed point theory, Cambridge University press, Cambridge (1990).

[13] Guerekata, G. M. A Cauchy problem for some fractional abstract differential equation with non local conditions, Nonlinear Analysis 70 (2009) 1873-1876.

[14] Liu. H. and Jiang. D. Two-point boundary value problem for first order implicit differential equations, Hiroshima Math.J.30(2000) 21-27.

[15] Ma. R. Existence and Uniqueness of Solutions to First - Order Three - Point Boundary Value Problems,Applied Mathematics Letters, 15(2002) 211-216.

${ }^{1}$ Department of Mathematics, Alexandria University, Alexandria, Egypt

E-mail address: amasayed@hotmail.com

${ }^{2}$ Department of Mathematics, Alexandria University, Alexandria, Egypt

E-mail address: emanhamdalla@hotmail.com

3 Department of Mathematics, Faculty of Science, Garyounis University, BengHAZI, LIBYA

E-mail address: k-welkadeky@yahoo.com 